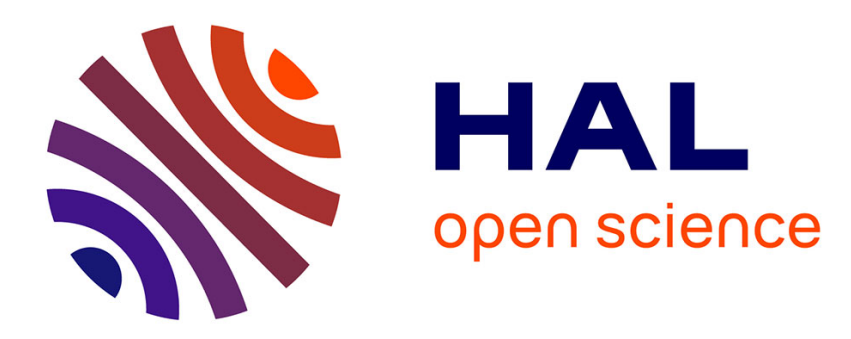

\title{
Evolution des peuplements de Pins maritimes dans le sud de la Bretagne
}

\author{
Jacques Lainé, X. Grenié
}

\section{To cite this version:}

Jacques Lainé, X. Grenié. Evolution des peuplements de Pins maritimes dans le sud de la Bretagne. Revue forestière française, 1990, 42 (4), pp.417-422. 10.4267/2042/26088 . hal-03425333

\section{HAL Id: hal-03425333 \\ https://hal.science/hal-03425333}

Submitted on 10 Nov 2021

HAL is a multi-disciplinary open access archive for the deposit and dissemination of scientific research documents, whether they are published or not. The documents may come from teaching and research institutions in France or abroad, or from public or private research centers.
L'archive ouverte pluridisciplinaire HAL, est destinée au dépôt et à la diffusion de documents scientifiques de niveau recherche, publiés ou non, émanant des établissements d'enseignement et de recherche français ou étrangers, des laboratoires publics ou privés. 


\title{
biologie et forêt
}

\section{ÉVOLUTION DES PEUPLEMENTS DE PINS MARITIMES DANS LE SUD DE LA BRETAGNE}

\author{
J. LAINÉ - X. GRENIÉ
}

\section{HISTORIQUE DU MILIEU}

Terre depuis longtemps occupée par l'homme, comme en témoignent de très nombreux mégalithes, le Sud de la Bretagne était couvert originellement d'une chênaie-hêtraie atlantique climacique, sauf au voisinage immédiat du littoral. D'après Le Thiec (1974), les premières traces de civilisation agraire remontent au Néolithique. L'atelier de Saint-Congard, de cette époque, révèle une population de chasseurs utilisant déjà de nombreux outils pour défricher. Les clairières, sommairement cultivées quelques années durant, étaient ensuite rapidement reconquises par la forêt. Avec l'augmentation de la population, les déboisements devinrent plus importants et plus durables. Cependant, au début du $I X^{e}$ siècle, ceuX-ci ne concernaient encore que quelques clairières. Arrivant d'Irlande et de Grande-Bretagne, les Bretons commencèrent le premier processus important de déforestation. Celui-ci fut poursuivi au $\mathrm{XII}^{\mathrm{e}}$ siècle par les moines cisterciens, puis du XVII au XIX ${ }^{e}$ siècle sous la pression de l'industrie et des forges.

Vers 1850 , la forêt ne recouvre plus que $5 \%$ du territoire. Près de la moitié de la surface restante occupée par l'agriculture était entretenue en "landes". Cette formation végétale basse, qui s'installe après abandon de la culture sur les sols primaires du Massif armoricain, est pauvre en espèces: Ajonc d'Europe, Ajonc nain, Bruyères cendrées et ciliées, Callune, Fougère aigle, Molinie, etc... avec des différences suivant les sols.

Cette lande jouait depuis longtemps un rôle important et indispensable dans la vie de la ferme bretonne, servant à la fois à l'alimentation du bétail et l'amendement des sols. Le mouton la parcourait toute l'année, consommant Ajoncs, Bruyères, Callunes et Agrostis. La petite vache 


\section{J. LAINÉ - X. GRENIÉ}

bretonne pie noire, ne pesant que $140 \mathrm{~kg}$ à l'âge adulte, consommait surtout l'Ajonc de mars à juin. L'hiver, l'Ajonc était pilé et servait de fourrage vert. Quand la végétation atteignait une certaine hauteur $(40 \mathrm{~cm}$ environ), l'agriculteur la coupait au ras du sol: c'était l'étrépage. Transportée dans l'étable, elle servait de litière au bétail. Une fois bien imbibée de matières organiques, cette litière était répandue sur les sols de culture dont elle était le seul amendement. Parfois dans les meilleures terres, après écobuage par le feu et épandage des cendres, on retravaillait le sol et on semait le seigle la première année, le sarrasin la deuxième, l'avoine la troisième, puis on laissait repartir le "landier". On allait même jusqu'à semer de l'Ajonc pour activer cette reprise. Cette lande "cultivée " faisait partie intégrante des exploitations agricoles de l'époque.

\section{PIN MARITIME SUR LANDE CULTIVÉE ET AGRICULTURE}

C'est sous Napoléon III et plus précisément sous l'influence de la Princesse Baciocchi, installée dans la région, que fut introduit le Pin maritime.

Les premières tentatives réalisées dans les forêts de Floranges et Camors furent couronnées de succès. Cette essence parfaitement adaptée au climat très ensoleillé, doux et humide de la région, se développa rapidement. Au moment des grandes restructurations foncières de la deuxième moitié du $X \mid X^{e}$ siècle, les propriétaires reboisent en Pin maritime quelques domaines cultivés. Mais c'est surtout sur les landes lorsque la terre est mise a nu par la pratique de l'étrépage, que cette essence très colonisatrice va s'installer par semis naturel.

L'agriculteur ne va pas s'opposer à ce développement, car sous le couvert léger du Pin, l'Ajonc continue à pousser, assurant toujours ses fonctions agricoles. Au moment de l'étrépage, tous les trois à cinq ans, l'agriculteur dépressera instinctivement les taches de semis trop denses, éliminera les mauvais sujets en conservant les meilleurs, car, ce bois qu'il appelle "sapin " se vend bien comme poteau de mine partant en Angleterre par les ports de Lorient, Vannes ou Redon, ou comme caisserie pour les poissonniers. Progressivement se constitueront ainsi des formations forestières irrégufières se régénérant naturellement grâce à l'étrépage qui permet aux graines le contact direct avec le sol. Peu à peu le Pin maritime devient un des éléments caractéristiques du paysage du Sud de la Bretagne et couvre 45000 hectares. Des vastes étendues de landes sont devenues des peuplements forestiers, régulièrement étrépés et parcourus par le bétail; ils sont pénétrables, irréguliers et vigoureux.

\section{PIN MARITIME ET ÉVOLUTION DE L'AGRICULTURE}

Vers 1955 l'intensification de l'agriculture apporte les amendements chimiques. La paille plus abondante remplace l'Ajonc dans les litières. Le bétail est nourri sur des pâtures cultivées ou avec des aliments spécialisés. La récolte de la lande ne devient plus nécessaire, elle est progressivement abandonnée d'autant qu'elle demande beaucoup de main-d'œuvre. La végétation de lande peut pousser sans limite et envahir les sous-bois de pins où l'agriculteur ne pénètre plus et n'intervient plus. Le sol n'étant plus dégagé, il n'y a presque plus de régénération naturelle et si la graine parvient au sol, le jeune plant est rapidement étouffé faute de dégagement. Après une coupe rase la repousse est très faible, en revanche après un incendie on peut voir de nombreux semis apparaitre et pousser convenablement. Et malheureusement ces peuplements non entretenus sont devenus très sensibles aux feux. Dans le Morbihan par exemple, département breton le plus concerné, 4000 hectares ont brûlé en 1976, 1000 en 1984 . Le sous-bois de lande dense et très développé explique l'importance de ces dégâts. Les chemins d'accès aux peuplements, que l'agriculteur n'utilise plus, sont souvent devenus impraticables et empêchent les services de DFCl d'accéder à l'origine des sinistres. 
D'autre part, les débouchés traditionnels du Pin maritime ont disparu; ceux-ci demandaient principalement des petits bois de qualité moyenne qui pouvaient être produits par une sylviculture rudimentaire, les peuplements étant exploités jeunes, entre 25 et 35 ans. Aujourd'hui, l'absence d'une sylviculture plus élaborée, permettant d'obtenir des bois de dimension répondant à la demande de l'industrie, a fermé les débouchés les plus rémunérateurs.

\section{LE PIN MARITIME AUJOURD'HUI}

Cette évolution écologique et économique des peuplements de Pin maritime, essence résineuse la plus importante en Bretagne par son volume, n'a pas échappé à la direction du Centre régional de la Propriété forestière (CRPF) qui a chargé Monsieur Colombet de réaliser une étude de typologie des stations dans cette zone. Celui-ci distingue deux grands types de peuplement: le premier, très peu représenté, installé dans d'anciens taillis simples:

"Les arbres sont généralement droits, bien élagués, avec un houppier important et bien équilibré, l'âge moyen avoisinant les soixante ans... Le volume de l'arbre moyen approche le mètre cube et permet d'obtenir des produits de qualité, utilisés en charpente, menuiserie ou déroulage ".

II s'agit en fait de taillis-sous-futaie. La réserve est constituée de 80 à 100 tiges de Pin maritime à l'hectare. La régénération en est mal assurée, le taillis, généralement de Châtaignier, très vigoureux, étant un concurrent redoutable des jeunes semis.

Le deuxième type de peuplement est installé sur d'anciennes landes:

"La densité est généralement trop forte, aucune éclaircie n'a été pratiquée... Les arbres sont flexueux, notamment sur parcelles à forte densité... Le volume de l'arbre moyen est faible 0,3 à $0.5 \mathrm{~m}^{3}$ pour un âge moyen de 35 à 40 ans... Les arbres exploités, mal conformés et de petit volume fournissent des produits de piètre qualité..."

Les peuplements sont très souvent incomplets. Leur qualité génétique paraît mauvaise, leur état sanitaire inquiétant (attaque d'Armillaire).

La différence de qualité entre ces deux peuplements s'expliquerait-elle par la qualité des sols ? Des observations très précises de F. Roussel, pédologue au CRPF, ont montré que cette hypothèse n'était pas la bonne, et que l'on pouvait observer ces deux types de peuplements de Pin maritime, des plus médiocres aux meilleurs, sur des sols dont les profils sont pratiquement identiques, sauf en ce qui concerne les humus.

Une étude de F. Toutain, directeur de recherches au CNRS de Nancy, et spécialiste des humus, a mis en évidence que, sur des sols de landes boisées en pins, les humus étaient de type mor, les horizons organo-minéraux sont très foncés, très épais avec une structure tassée. La microfaune est très peu active et presque inexistante. Les boulettes fécales d'enchytréïdes et de microarthropodes traduisent la difficulté d'humidification. Les lombrics ne sont pas décelables de même que les champignons de pourriture blanche. L'activité biologique est très faible. L'humus ne remplit plus le rôle essentiel de transformation de la matière organique et de transfert de sels minéraux. La pratique régulière de l'étrépage pendant des siècles, par exportation de la matière organique, explique cette désaturation des horizons superficiels et l'acidification importante probablement aggravée par la végétation de lande (Bruyères, Pins).

La dégradation évidente de ces peuplements de pins serait donc due au blocage de l'activité biologique du milieu sur lequel ils sont implantés. II a été proposé de qualifier d'“ effet lande " cette action dépressive ainsi mise en lumière. L'absence de sylviculture pourrait être un autre élément de dégradation, mais le fait que d'autres peuplements d'essences exotiques, convena- 
blement entretenus, installés sur ces mêmes milieux aient échoué, infirme cette hypothèse et confirme l'effet lande. Celui-ci n'est qu'une des formes de l'antécédent cultural résultant d'une action anthropique très marquée. Quand cette action, qui est une intervention agricole traditionnelle (pâturage, étrépage), a cessé depuis un certain temps, on peut observer dans de nombreuses stations sur sol sain et relativement profond, l'apparition progressive sous le couvert des Pins d'un sous-bois feuillu, de Chênes pédonculés, Châtaigniers, Bouleaux et parfois Merisiers, formant çà et là des bouquets sous lesquels la composition de la flore évolue également (lierre, ronces, etc ...). On retrouve donc certaines espèces de la forêt d'origine. Ces peuplements de pins abandonnés à eux-mêmes devraient évoluer lentement vers une formation forestière de type climacique.

\section{PERSPECTIVES D'AVENIR}

Dans l'état actuel des choses, dans le Sud de la Bretagne, le caractère artificiel des peuplements de pins maritimes installés sur landes, leur absence de pérennité, les risques particuliers d'incendie qu'ils présentent, leur état sanitaire préoccupant et enfin leur très faible rentabilité, posent aux forestiers de nombreux problemes. Excluons de ces observations les peuplements installés dans d'anciens taillis ou mélangés avec d'autres feuillus qui peuvent fournir des bois de qualité. Ce type de sylviculture mérite d'être conservé et peut être amélioré.

Peut-on envisager pour les autres peuplements une sylviculture intensive? Sur ces milieux d'ancienne lande, fortement dégradés par leur passé cultural, où les introductions d'autres essences résineuses ont également souvent échoué, il parait indispensable d'essayer de modifier préalablement le milieu.

Que dit F. Toutain :

"Afin de redonner aux sols de lande des potentialités forestières acceptables, il faut stimuler l'activité biologique qui fait défaut et celle des vers de terre en effectuant les opérations suivantes :

- apporter du calcium et divers éléments minéraux à ces sols pauvres ;

- effectuer des travaux du sol (cover-crop) afin d'accélérer le recyclage de la matière organique ;

- pratiquer le plus possible dans le cas de plantations le mélange d'essences qui assurera une certaine activation de la vie biologique;

- favoriser, dans la mesure du possible, les plantations d'essences améliorantes, feuillus notamment ;

- tenter des essais :

- de plants prémycorhizés susceptibles de faciliter la nutrition minérale,

- d'introduction de vers de terre annéciques après apports d'éléments minéraux divers".

Nous voyons que ces propositions ne vont guère dans le sens d'une mono-sylviculture du Pin maritime sur ces milieux. Ajoutons que, pour ce pin, le risque d'incendie restera majeur sans une destruction permanente très onéreuse de la végétation du sous-bois qu'il engendre. Cependant il faut bien sûr essayer de conduire à terme d'exploitation les peuplements existants, susceptibles de fournir une production appréciable en quantité et qualité. Mais après une coupe rase, le choix d'autres essences paraît souhaitable, car sur le plan économique, le caractère de la forêt bretonne de faible étendue, morcelée, dispersée, loin des centres de transformation, ne paraît pas se prêter à la gestion d'une essence fortement concurrencée au niveau mondial par des arbres très comparables, comme par exemple le remarquable "Pinus taeda" dans des pays de grande surface et de potentialités forestières très supérieures. 


\section{J. LAINÉ - X. GRENIÉ}

L'étude de typologie de M. Colombet, dirigée par le CRPF de Bretagne, mentionnée plus haut, avait pour but de proposer aux propriétaires forestiers, tout au moins sur certains milieux bien définis et faciles à reconnaître, un choix d'essences plus productives et plus résistantes au feu, susceptibles de remplacer le Pin maritime.

L'application du catalogue des stations, déjà commencée sur le terrain, avec l'aide du Conseil général du Morbihan et de la Direction départementale de l'Agriculture et de la Forêt (DDAF), devrait permettre de réaliser un aménagement aussi rationnel que possible de ces difficiles milieux d'anciennes landes. En tout état de cause le choix devra tenir compte impérativement du passé cultural; et la sylviculture adoptée devra essayer de résoudre ce grave problème de la dégradation des sols. D'ailleurs toute sylviculture ne devrait-elle pas ménager les sols, voire les reconstituer en assurant les équilibres biologiques indispensables?

\begin{tabular}{|c|} 
J. LAINÉ \\
CENTRE D'ÉTUDES TECHNIQUES FORESTIÉRES DU MORBIHAN \\
Boulevard de la Résistance \\
BP 398 \\
56009 VANNES CEDEX \\
CENTRE RĖGIONAL DE LA PROPRIĖTÉ FORESTIĖRE \\
DE BRETAGNE \\
8, place dU COlOMbIer \\
35000 RENNES \\
\hline
\end{tabular}

\section{BIBLIOGRAPHIE}

COLOMBET (M.). - Les Landes de Lanvaux - Types de station. Performances des principaux résineux utilisés en reboisement. - Étude réalisée par le Centre régional de la Propriété forestière de Bretagne, 1988. $190 \mathrm{p}$.

LE THIEC (M.). - Contribution à l'étude des Landes de Lanvaux. Rôle de l'action de l'homme sur leur état actuel et perspective d'avenir. - ENSA - Université de Rennes, 1974. - $61 \mathrm{p}$.

MAURICE (L.). - Écosystème des landes bretonnes - Caractérisation des landes - évaluation - problèmes des landes littorales - influences humaines - historique. - Saint-Brieuc : Centre départemental de Documentation pédagogique des Côtes-du-Nord, 1988. - 91 p. (Collection d'Écologie armoricaine).

RIGOLOT (E.). - Le Problème des incendies dans les landes de Lanvaux. Étude préalable et propositions. Rennes: Centre régional de la Propriété forestière de Bretagne, 1987. - $94 \mathrm{p}$.

ROUSSEL (F.). - Caractérisation des sols en milieu forestier dans le Massif armoricain. - 2 tomes. Rennes: Centre régional de la Propriété forestière de Bretagne, 1983. - $30 \mathrm{p}$.

TOUTAIN (F.). - Les Humus forestiers. Structure et mode de fonctionnement. - Revue forestière française, vol. XXXIII, nº, 1981 , pp. 449-462.

TOUTAIN (F.). - Biodynamique des humus dans les landes de Lanvaux. - Rapport de mission pour le Centre régional de la Propriété forestière de Bretagne, 1988. - $10 \mathrm{p}$. + photos. 